\title{
A Study of Feature Stability of Contact-Less Imaging Based on Palm Vein
}

\author{
Weiqi Yuan ${ }^{1}$, Wei Wu ${ }^{1,2}$, Lantao Jing ${ }^{1}$, Deqi Kong ${ }^{1}$, Lili Wang ${ }^{1}$ \\ ${ }^{1}$ Computer Vision Group, Shenyang University of Technology, Shenyang, China \\ ${ }^{2}$ Information Engineering, Shenyang University, Shenyang, China \\ Email: yuan60@126.com, wuwei4296@163.com
}

Received 2013

\begin{abstract}
Palm vein hidden under the skin and its distribution feature is hard to be stolen, which makes the palm vein recognition to be a high security biometric authentication method. Contact-less palm vein imaging can avoid the spread of disease, thus expanding the application range of palm vein biometric authentication devices. However, due to the different understanding of the right imaging position and the change of fingers open degree, contact-less palm vein image acquisition led to a certain degree of translation, rotation, scaling and shear, that is, the image deformation. Image deformation causes the imaging feature unstable. In this paper, the effect of image deformation to the stability of palm vein features is studied by some similarity parameters. First, feature points in the palm were marked, contact-less imaging and contact imaging of palm vein were acquired. Then, this paper calculated the similarity parameters of the contact-less imaging to contact imaging and gave corresponding analysis. Experimental results show that contact-less palm vein imaging was stable, and derived the linear regression equation of relationship between sample space and the recognition rate: $\mathrm{y}=$ $-0.000903 x+1.0332$, coefficient of determination $\mathrm{R}^{2}=0.9824$. This research provided effective and detailed data to the study of contact-less palm vein recognition and gave powerful support to contact-less multi-feature fusion recognition based on hand.
\end{abstract}

Keywords: Palm Vein; Contact-Less Imaging; Stability

\section{Introduction}

Palm vein is a permanence and uniqueness physiological feature of human [1,2]. Palm vein recognition as a new family of biometric technology has gained more and more attentions these years and it is expected to have a wide range of security application. From the health perspective, contact palm vein imaging may cause the spread of germs, resulting in resistance to some users. This problem is particularly exacerbated during the outbreak of epidemics or pandemics like SARS and Influenza A (H1N1) which can be spread by touching germs leftover on surfaces. In the outdoor environment, this contact palm vein device easily contaminated, affected its use in the access control system. Contact imaging brings inconvenience in actual use, which makes the developing contact-less palm vein recognition instruments become new direction. The "contact-less" in this research means no guidance pegs were used to constraint the position of the hand. The user's hand can face the sensor naturally as prompt during image acquisition. Obviously this contact-less imaging can avoid the spread of germs and improves ease of use.

Like the other biometric technology, the contact-less palm vein recognition in this paper includes two stages: registration stage and recognition stage. In registration stage, there are three steps: preprocessing the captured image firstly, then feature extraction and saving the feature to database for further matching lastly. In recognition stage, the captured image also taken the stage of preprocessing, feature extraction then matches the feature of the palm vein image in the database. Whether the two images belong to the same hand was judged by the similarity of the feature extracting from two images. Feature extracting is the most important. At present, palm vein feature extraction method can be roughly divided into the following two categories: structure-based feature extraction, such as point features, line features; spatial frequency domain transform based feature extraction, such as the wavelet transform. Point feature extraction is a simple way of extracting crossover point, bifurcation point or endpoint of blood vessels in the palm image (as shown in Figure 1). Literature [3-5] adopt this feature extraction method. The deformation caused by contact-less imaging will be reflected in the change of spatial location of these feature points. This paper takes the crossover point, bifurcation point or endpoint of blood 

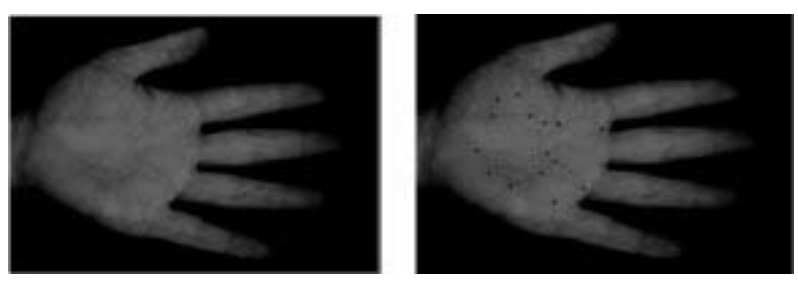

(a)

(b)

Figure 1. NIR image of palm vein and feature point mark. (a) NIR image of palm vein; (b) bifurcation point and ending point mark.

vessels in the palm image as the feature points. The paper represents palm vein image deformation with the change of these points in the space position.

To achieve the contact-less palm vein recognition, the feature stability of palm vein is the question must be discussed firstly.

Some other literature inferred "contact-less imaging" also. Reference [6] captured the finger vein contact-less with transmission illumination method, while our capture method is reflection illumination method. Reference [7] just mentioned "contact-less image", but not clearly expressed how to realize. The contact-less imaging realized in [8] used capture window, so it was not really contactless method. Reference [9] realized contact-less palm vein recognition with a robust processing method, however, it didn't involve the feature stability of imaging. To the best of our knowledge, there is scant research which focuses on the study of feature stability of contact-less image based on palm vein.

For fixed capture distance and illumination angle, contact imaging can guarantee for feature stable of palm vein image. If the contact-less imaging is more similar to contact image, the contact-less image is more stable. At the same time, if the inter-class distinction between images is bigger, the recognition rate is higher. To investigate the feature stability of palm vein image, this paper designs two experiments to evaluate the similarity parameter and recognition rate.

\section{The Evaluation Methodology of Feature Stability of Contact Image Based on Palm Vein}

We assume that the hands just take rigidity shape change during the acquisition process.

If extracted all feature points in palm vein image by programming, the result would be affected a lot by the algorithm of preprocessing, feature extraction and feature matching. To eliminate this effect, our research designed an experiment to measure the similarity of contact-less image to contact image. Additionally, palm vein image quality is not good enough for every person. Extracting feature points in this picture are difficult and are not ac- curate enough. Actually, we want to get the offset of the contact-less imaging to contact imaging, rather than the actual location of feature points. Therefore, in order to remove these impacts, this paper presents a simulation of the palm vein feature point method, and accordingly designed two experiments.

\subsection{Evaluation Methods Based on Similarity}

Contact-less imaging not only might lead to translation and rotation, but also scaling and shear. The scaling is caused by the different distance to sensor and the shear is caused by the angle to sensor. The shear include horizontal shear and vertical shear [10], shown in Figure 2. Contact imaging might lead to translation and rotation. Translation and rotation can be adjusted by the algorithm.

The effect on images led to the change of position of the point in palm vein. The similarity of two images can be measured by the change of position of feature points in two images. Euclidean distance is one of the most simplest and effective algorithms in measurement of the similarity of two images. This research measured the change of position of feature points in contact-less image to position of feature points in contact image by Euclidean distance. The similarity of contact-less image to contact image was represented by this change.

We figured 9 feature points in the hands of volunteers to represent the feature of the image of palm vein. The position of feature point has no feature of vein; it only represents the position of the point that meets some feature condition. In this experiment, the feature points were just the constant points of palm vein. They represented the bifurcation point and ending point on the palm vein. The stability of these 9 feature points represented the stability of the whole image. The change of these 9 feature points in contact-less image to contact image represented the similarity of the two images. The 9 points were specified as shown in Figure 2.

First, we drew vertical line from the heel of thumb and drew midline from middle finger, these two lines jointed in the point marked point 5 . Then, we drew a square of 4 $\mathrm{cm}$ length of sides with the central point was point 5 and the coordinate was the heel of thumb and midline from middle finger. Last, we marked the peak of square with

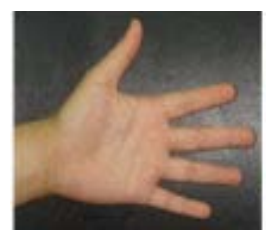

(a)

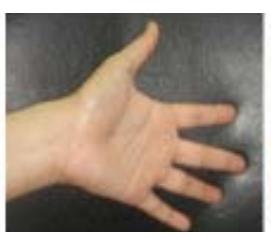

(b)

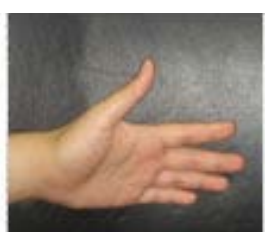

(c)
Figure 2. Level imaging and shear imaging. (a) Level imaging; (b) Vertical shear imaging; (c) Level shear imaging. 
point 1 , point 3 , point 9 , point 7 and marked the middle point of every length with point 2 , point 6 , point 8 , point 4. We registered contact-less image to contact image with point 5 . After wiped off the deviation of rotary and translation from contact-less image to contact image, the other 8 points could represent the change from every direction.

Experiment method: Every volunteer took one contact image of palm vein, three contactless images of palm vein. After wiped off the deviation of rotary and translation from contact-less image to contact image, we called it registration image. We computed Euclidean distance of 9 points in registration image to 9 points in contact image. This Euclidean distance could represent the similarity of contact-less image to contact image.

As shown in Figure 3, the coordinates of 9 character points in contact image were $\operatorname{dot}_{1}\left(\mathrm{x}_{1}, \mathrm{y}_{1}\right), \operatorname{dot}_{2}\left(\mathrm{x}_{2}, \mathrm{y}_{2}\right)$, $\operatorname{dot}_{3}\left(\mathrm{x}_{3}, \mathrm{y}_{3}\right), \operatorname{dot}_{4}\left(\mathrm{x}_{4}, \mathrm{y}_{4}\right), \operatorname{dot}_{5}\left(\mathrm{x}_{5}, \mathrm{y}_{5}\right), \operatorname{dot}_{6}\left(\mathrm{x}_{6}, \mathrm{y}_{6}\right), \operatorname{dot}_{7}\left(\mathrm{x}_{7}, \mathrm{y}_{7}\right)$, $\operatorname{dot}_{8}\left(\mathrm{x}_{8}, \mathrm{y}_{8}\right), \operatorname{dot}_{9}\left(\mathrm{x}_{9}, \mathrm{y}_{9}\right)$. The coordinates of 9 character points in registration image were $\operatorname{dot}_{1}{ }^{\prime}\left(\mathrm{x}_{1}{ }^{\prime}, \mathrm{y}_{1}{ }^{\prime}\right), \operatorname{dot}_{2}{ }^{\prime}$ $\left(\mathrm{x}_{2}{ }^{\prime}, \mathrm{y}_{2}{ }^{\prime}\right), \operatorname{dot}_{3}{ }^{\prime}\left(\mathrm{x}_{3}{ }^{\prime}, \mathrm{y}_{3}{ }^{\prime}\right), \operatorname{dot}_{4}{ }^{\prime}\left(\mathrm{x}_{4}{ }^{\prime}, \mathrm{y}_{4}{ }^{\prime}\right), \operatorname{dot}_{5}{ }^{\prime}\left(\mathrm{x}_{5}{ }^{\prime}, \mathrm{y}_{5}{ }^{\prime}\right)$, $\operatorname{dot}_{6}{ }^{\prime}\left(\mathrm{x}_{6}{ }^{\prime}, \mathrm{y}_{6}{ }^{\prime}\right), \operatorname{dot}_{7}{ }^{\prime}\left(\mathrm{x}_{7}{ }^{\prime}, \mathrm{y}_{7}{ }^{\prime}\right), \operatorname{dot}_{8}{ }^{\prime}\left(\mathrm{x}_{8}{ }^{\prime}, \mathrm{y}_{8}{ }^{\prime}\right), \operatorname{dot}_{9}\left(\mathrm{x}_{9}{ }^{\prime}, \mathrm{y}_{9}{ }^{\prime}\right)$.

We marked Euclidean distance of 9 points in registration image to the 9 points in contact image with sum. The equation of sum was showed as (1).

$$
\text { sum }=\sqrt{\sum_{i=1}^{i=9}\left(\left(x_{i}-x_{i}^{\prime}\right)^{2}+\left(y_{i}-y_{i}^{\prime}\right)^{2}\right)}
$$

The similarity of contact-less image to contact image was measured by the average value of Euclidean distance of 3 registration images to contact image.

According to Equation (2), we calculate the mean value of 3 Euclidean distances of registration imaging to contact imaging. This mean value measured the similarity of contact-less imaging and contact imaging by this experimenter. Then we calculate the changes of distance of point 5 to point $1,2,3,4,6,7,8,9$ separately. That is the offset of 8 directions as Figure 4.

$$
\text { ave }=\frac{\sum_{n=1}^{n=3} \operatorname{sum}(n)}{3}
$$

\subsection{Evaluation Methods Based on Recognition Rate}

This paper used recognition rate as another index to measure contact-less imaging characteristics stability. More significant the difference between the classes, (the higher the recognition rate), and the more suitable for subsequent identification.

In this experiment, we also draw 9 points on the palm of experimenter to simulate the palm veins feature points. It had difference with the first experiment. The length and width of rectangle enclosed by point 1 , point 2 , point

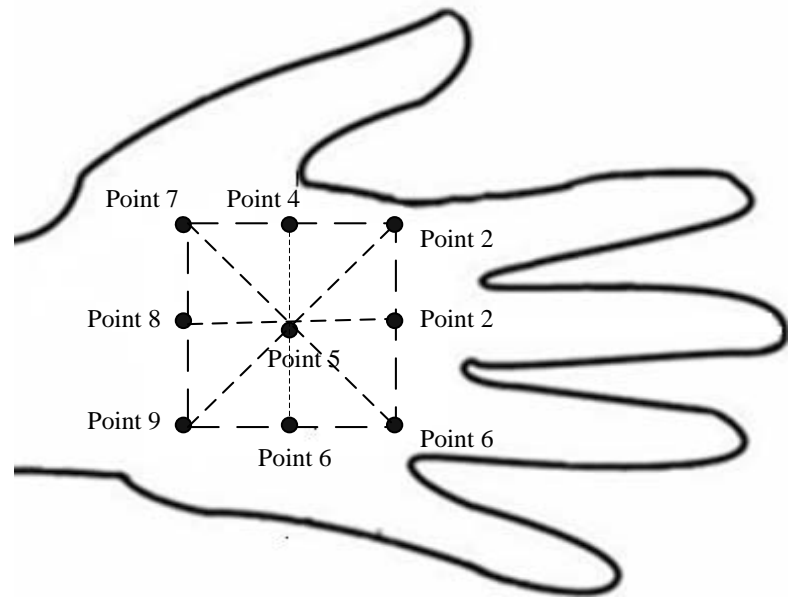

Figure 3.9 characteristic points on palm vein in experiment 1.

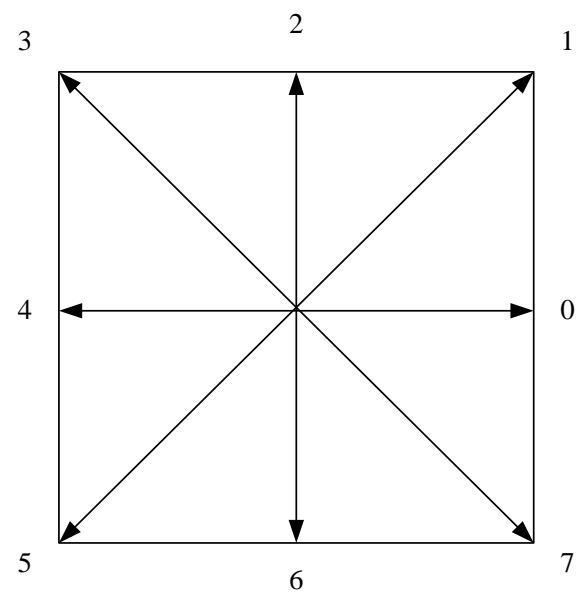

Figure 4. Schematic diagram of 8chain code.

3 , point 6 , point 7 , point 8 , point 9 , and point 4 were not regular. $\mathrm{T}$ he second difference was the location of point 5 only enclosed in the rectangle, rather than the center, the location of the nine points shown in Figure 5.

Every experiment took a contact imaging as the registration, extracted the coordinates of night points of this image and stored them in the sample database. We calculated the degree of angle $\alpha$, shown in Figure 6 .

The equation was following.

$$
\alpha=\arccos \frac{b^{2}+c^{2}-a^{2}}{2 b c}
$$

The degree of the angle $\alpha$ is also stored in the sample database. The degree of the angle $\alpha$ is also stored in the sample database. We took contact-less image of every experiment as the login image. Extract the degree of angle $\alpha$, and nine coordinates of feature points as the match data.

The first step was to determine with angle $\alpha$, chose images with angle of $\alpha \pm 1^{\circ}$ into the next match, select 


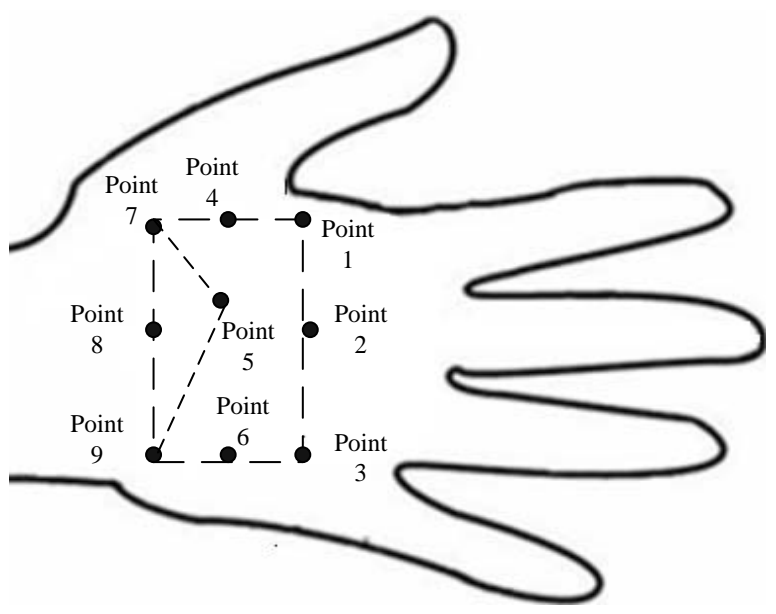

Figure 5.9 characteristic points on palm vein in experiment 2.

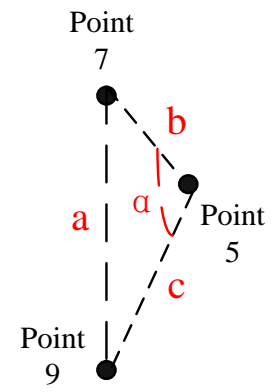

Figure 6. Schematic diagram of angle $\alpha$.

minimum Euclidean distance of 9 feature points to determine the same person, otherwise identified as different people. Recognition rate (Recognition Rate, RR) is calculated as (4) [11]:

$$
R R=\left(1-\frac{N F R+N F A}{N A A+N I A}\right) \times 100 \%
$$

NAA and NIA were the attempt times of the legitimate user and impostor in (4). NFR and NFA are times of the false rejection and false acceptance. Programmatically accomplish the above calculation.

This paper calculated the number of samples with 30, $60,90,120,150,180$. With this change of samples the regular pattern could be caught.

\section{Experiment and Result Analysis}

\subsection{Similarity Experiment}

To ensure the reliability of experimental data, there were 180 volunteers participating in the experiment, including 90 women. 180 volunteers aged 20 to 40 years old. The experimenter divided volunteers into six groups of 30 people, including 15 female, each group is to meet a minimum sample space.

In our experiment, contact images were captured in the condition of volunteer's hand placed on the bracket. The bracket indirect under the sensor of image and the distance was $10 \mathrm{~cm}$. Contact-less images were captured in the condition of volunteers placed there hand under the sensor of image $10 \mathrm{~cm}$, parallel his/her hand with the sensor. The five fingers of the volunteer separate naturally. The image resolution was $1280 \times 960$ pixels of two kinds of images.

First of all, Figured 9 character points on the left hand of every volunteer, just like the Figure 2 showed. Secondly, captured 1 image of each volunteer with contact pattern and named images with $A_{n}(n=1 \cdots 180)$. Then, captured 3 images of each volunteer with contact-less pattern and named them with $B_{n 1}, B_{n 2}, B_{n 3}(n=1 \cdots 180)$ separately.

1) Step 1: Extracted coordinate of 9 character points of contact image $A_{1}$ and coordinate of 9 character points of contact-less image $B_{11}$ separately. That were point sets of $a_{1}$ and $b_{11}$.

2) Step 2: We accomplished registration from image $B_{11}$ to image $A_{1}$. Coincide the coordinate of 5 th point in image $B_{11}$ with image $A_{1}$. Adjusted the middle finger of image $B_{11}$ to the middle finger of image $A_{1}$.

3) Step 3: In the registration image $B_{11}$, the coordinates of 9 points maked up the point set $c_{11}$.

4) Step 4: We computed the Euclidean distance of the correspond point in point sets of $c_{11}$ and the point in point set $a_{1}$. Now we computed the 9 Euclidean distance of 9 character to sum 1 .

5) Step 5: Followed step 1-4, settle contact-less image $B_{12}, B_{13}$ separately, then we get sum 2 , sum s. $_{\text {. }}$.

6) Step 6: Ave $_{1}$ represents the average value of 3 Euclidean distance of 9 points in registration image to 9 points in contact image. The equation of sum is showed as (2).

7) Step 7: In accordance with this method, the remaining 29 samples were completed. We got ave1 ave30.

8) Step 8: The remaining five groups of samples were calculated and we got the data.

\subsection{Result Analysis of Similarity Experiment}

To reflect the variability of 6 sets of data, we calculated a number of parameters in Table 1, including the sample mean, the sample variance, the sample standard deviation and range, the equations as following:

Sample mean:

$$
\bar{x}=\frac{1}{n} \sum_{i=1}^{n} x_{i}
$$

Sample variance:

$$
s^{2}=\frac{1}{n-1} \sum_{i=1}^{n}\left(x_{i}-\bar{x}\right)^{2}
$$


Table 1. Similarity statistical parameters of 6 group sample.

\begin{tabular}{cccccc}
\hline Feature & $\begin{array}{c}\text { Sample } \\
\text { Mean }\end{array}$ & $\begin{array}{c}\text { Sample } \\
\text { Median }\end{array}$ & $\begin{array}{c}\text { Sample } \\
\text { Variance }\end{array}$ & $\begin{array}{c}\text { Sample } \\
\text { Standard } \\
\text { Deviation }\end{array}$ & $\begin{array}{c}\text { Sample } \\
\text { Utmost }\end{array}$ \\
\hline 1 & 106.95 & 97.56 & 1883.28 & 43.40 & 186.81 \\
2 & 107.93 & 97.38 & 1410.37 & 37.55 & 171.52 \\
3 & 99.12 & 91.78 & 1538.47 & 39.22 & 166.49 \\
4 & 99.77 & 99.77 & 1755.89 & 41.90 & 158.70 \\
5 & 99.84 & 92.64 & 2173.68 & 46.62 & 192.63 \\
6 & 97.73 & 89.85 & 1406.10 & 37.50 & 149.96 \\
\hline
\end{tabular}

Sample standard deviation:

$$
s=\sqrt{\frac{1}{n-1} \sum_{i=1}^{n}\left(x_{i}-\bar{x}\right)^{2}}
$$

Sample mean in Table 1 varied between 97.73 107.93, varied in 10.2 pixels. Sample median between the range of 89.85 - 99.77, varied in 10.2 pixels. That means the sample concentration was good.

Sample variance was a way to reflect the data variability of statistical parameters, the larger the sample variance, the data demonstrate the variability of the group was greater. In the six groups, the sample variance and standard deviation of the fifth group were maximum, indicating some of experimenters in this group had taken too far or too near distance from requirements, see the original picture, we found that two experimenters took too far distance from the lens, resulting in the median of the group was small, but the sample variance, sample standard deviation and sample utmost were great. The same situation happened in the first group. Other groups of data indicates the majority of the experimenter could understand contact-less imaging probably accurate and the way of contact-less imaging was basically stable.

Directions offset of six groups data was shown in Table 2. In order to analyze the above six groups of data, we draw the surface figure as shown in Figure 7.

In the figure, the $\mathrm{x}$-coordinate was 8 directions, the $y$-coordinate was offset and the unit was pixels. The three-dimensional was six groups of data in the direction of distribution. Through Figure 7, we could draw a conclusion of offset in direction 5-1, 5-3, 5-7, 5-9 were larger than the other four directions. In the direction 5-3 and $5-9$, we got the maximum of offset.

\subsection{Result Analysis of Recognition Rate Experiment}

In the sample space of 30 people, we matched contactless image and contact image of every experimenter. Experiments conducted in-class match 30 times, interclass match $C_{60}^{2}=1770$ times, recognition rate of $99.84 \%$. The remaining five recognition rates of the sample space
Table 2. basic statistical parameters of 8 directions offset (unit: pixel).

\begin{tabular}{ccccccccc}
\hline Direction & $\mathbf{5 - 1}$ & $\mathbf{5 - 2}$ & $\mathbf{5 - 3}$ & $\mathbf{5 - 4}$ & $\mathbf{5 - 6}$ & $\mathbf{5 - 7}$ & $\mathbf{5 - 8}$ & $\mathbf{5 - 9}$ \\
\hline 1 & 35.1 & 28.3 & 37.2 & 25.9 & 30.2 & 38.6 & 30.5 & 41.0 \\
2 & 32.4 & 20.4 & 33.7 & 27.8 & 29.2 & 31.2 & 25.4 & 35.8 \\
3 & 35.0 & 27.6 & 36.1 & 24.1 & 26.0 & 31.5 & 26.1 & 36.5 \\
4 & 33.9 & 24.3 & 32.5 & 23.4 & 23.8 & 32.2 & 26.6 & 36.4 \\
5 & 32.7 & 23.9 & 37.5 & 24.7 & 25.4 & 38.5 & 28.1 & 34.2 \\
6 & 32.5 & 25.8 & 33.1 & 24.5 & 22.5 & 34.0 & 25.2 & 31.0 \\
mean & 33.6 & 25.1 & 35.0 & 25.1 & 26.2 & 34.3 & 27.0 & 35.8 \\
\hline
\end{tabular}

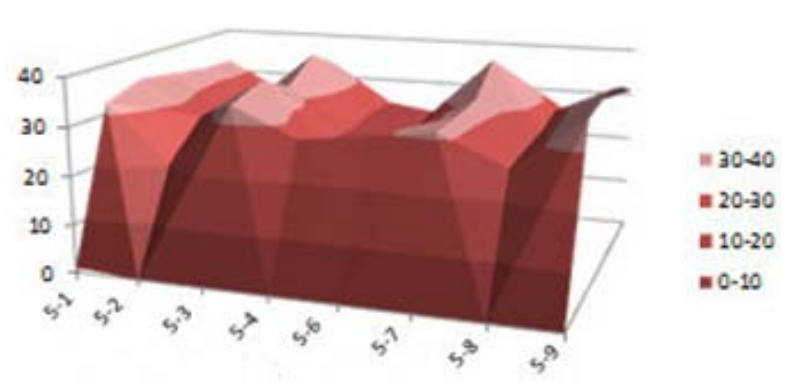

Figure 7. 8 direction offset contrast.

Table 3. Relationship between sample space and recognition rate.

\begin{tabular}{lcccccc}
\hline $\begin{array}{l}\text { Sample } \\
\text { Space }\end{array}$ & $\begin{array}{c}\mathbf{3 0} \\
\text { person }\end{array}$ & $\begin{array}{c}\mathbf{6 0} \\
\text { person }\end{array}$ & $\begin{array}{c}\mathbf{9 0} \\
\text { person }\end{array}$ & $\begin{array}{c}\mathbf{1 2 0} \\
\text { person }\end{array}$ & $\begin{array}{c}\mathbf{1 5 0} \\
\text { person }\end{array}$ & $\begin{array}{c}\mathbf{1 8 0} \\
\text { person }\end{array}$ \\
\hline $\begin{array}{l}\text { Recognition } \\
\text { Rate }\end{array}$ & $99.8 \%$ & $98.1 \%$ & $95.8 \%$ & $92.8 \%$ & $90.3 \%$ & $86.1 \%$ \\
\hline
\end{tabular}

as shown in Table 3.

We did linear regression analysis from TABLEIII and got the trend line of relationship between sample space and recognition rate, as shown in Figure 8.

At last, we derived the linear regression equation of relationship between sample space and the recognition rate: $\mathrm{y}=-0.000903 \mathrm{x}+1.0332$, the judge coefficient was $\mathrm{R}^{2}=0.9824$. Coefficient of determination $\mathrm{R}^{2}=0.9824$ indicates the explanation power of the regression modal, that means sample could explained recognition rate diversity $98.24 \%$.

\section{Conclusions}

In this paper, we measured similarity of the contact-less image to contact image, drew the conclusion of contact-less imaging was basically stable. Scaling and shear had effect on contact-less image stability. In the 8 direction of chain code, offset of 5-3 direction and 5-9 direction were the largest. This paper also derived the linear regression equation of relationship between sample space and the contact-less recognition rate in our imaging con- 


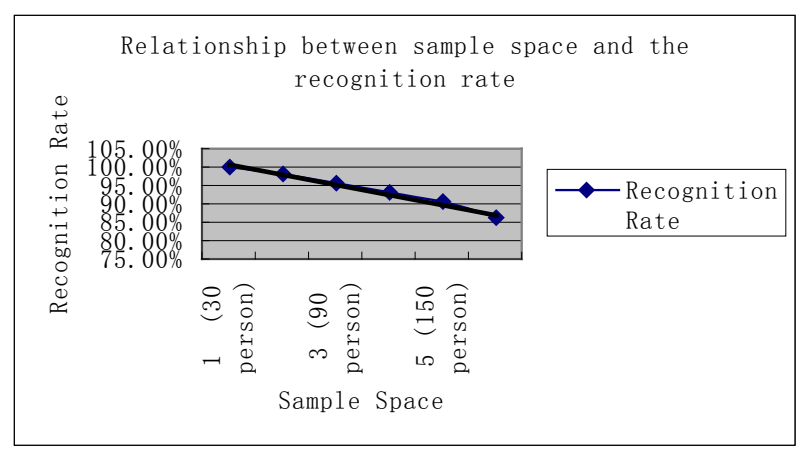

Figure 8. Trend line of relationship between sample space and recognition rate.

dition. The deviation of contact-less imaging was caused by the different understanding of imaging situation. The result we got from this research provided effective and detailed data to the study of contact-less palm vein recognition and gave powerful support to contact-less hand multi-feature fusion recognition.

In our future research, we will try to give the curve of high scope and angle scope that fit for contact-less imaging. It will support the contact-less palm vein recognition and the fusion with the other biometrics feature. At the same time, the curve of high scope and angle scope that fit for contact-less imaging will benefit for the other contact-less biometric technologies, such as hand vein recognition and palm print recognition.

\section{Acknowledgements}

This work is supported by National Natural Science
Foundation of China (60972123), Specialized Research Fund for the Doctoral Program of Higher Education (20092102110002) and Shenyang Science and Technology Development Program (F10-213-1-00).

\section{References}

[1] A. Jain, R. Bolle and S. Pankanti, "Biometrics: Personal Identification in Networked Society,” Kluwer Academic Publishers, Dordrecht, 1999. http://dx.doi.org/10.1007/b117227

[2] M. Watanabe, "Palm Vein Authentication," Advances in Biometrics, Springer, Berlin, 2008.

[3] B. Prasanalakshmi and A. Kannammal, "A Secure Cryptosystem from Palm Vein Biometrics in Smart Card," The 2nd International Conference on Computer and Automation Engineering (ICCAE), 26-28 February 2010, pp. 653-657.

[4] Y. Z. Chao, Q. P. Guo and X. L. Lian, "Algorithm Research of Vein Recognition Based on Feature Point," Computer and Digital Engineering, Vol. 36, No. 5, 2008, pp. 1-3.

[5] L. Wang, G. Leedham and D. S.-Y. Cho, "Minutiae FeaTure Analysis for Infrared Hand Vein Pattern Biometrics,” Pattern Recognition, Vol. 41, No. 3, 2008, pp. 920-929. http://dx.doi.org/10.1016/j.patcog.2007.07.012

[6] S. Hero, "Vein Imaging Device, Vein Imaging Method and Vein Recognition Device," China, Application for Patent for Invention 200910168806.0, 2010.03.03. 